\title{
Resource allocation with interference mitigation in OFDMA femtocells for co-channel deployment ${ }^{\mathrm{a}}$
}

\author{
Haijun Zhang ${ }^{1} 2^{*}$, Xiaoli Chu ${ }^{3}$, Wenmin $\mathrm{Ma}^{1}$, Wei Zheng ${ }^{1}$ and Xiangming Wen ${ }^{1}$
}

\begin{abstract}
Femtocells have been considered as a promising technology to provide better indoor coverage and spatial reuse gains. However, the co-channel deployment of macrocells and femtocells is still facing challenges arising from potentially severe inter-cell interference. In this article, we investigate the uplink resource allocation problem of femtocells in co-channel deployment with macrocells. We first model the uplink power and subchannel allocation in femtocells as a non-cooperative game, where inter-cell interference is taken into account in maximizing the femtocell capacity and uplink femto-to-macro interference is alleviated by charging each femto user a price proportional to the interference that it causes to the macrocell. Based on the non-cooperative game, we then devise a semi-distributed algorithm for each femtocell to first assign subchannels to femto users and then allocate power to subchannels. Simulation results show that the proposed interference-aware femtocell uplink resource allocation algorithm is able to provide improved capacities for not only femtocells, but also the macrocell, as well as comparable or even better tiered fairness in the two-tier network, as compared with existing unpriced subchannel assignment algorithm and modified iterative water filling-based power allocation algorithm.
\end{abstract}

\section{Introduction}

Nowadays above $50 \%$ of voice services and $70 \%$ of data traffics occur indoors [1]. Insufficient indoor coverage of macrocells has led to increasing interest in femtocells, which have been considered in major wireless communication standards such as 3GPP LTE/LTE-Advanced [2]. Dedicated-channel deployment of femtocells, where femtocells and macrocells are assigned with different (or orthogonal) frequency bands, may not be preferred by operators due to the scarcity of spectrum resources and difficulties in implementation. While in co-channel deployment, where femtocells and macrocells share the same spectrum, cross-tier interference could be severe [3], especially when femtocell base stations (FBSs) are deployed close to a macrocell base station (MBS) [4]. Due to the fundamental role of macrocells in providing blanket

\footnotetext{
${ }^{*}$ Correspondence: zhj@bupt.edu.cn

1 Beijing Key Laboratory of Network System Architecture and Convergence, Beijing University of Posts and Telecommunications, Beijing 100876, China 2 Institute of Telecommunications, King's College London, London WC2R 2LS, UK Full list of author information is available at the end of the article
}

cellular coverage, their capacities and coverage should not be affected by co-channel deployment of femtocells.

Power control has widely been used to mitigate intercell interference in co-channel deployment of femtocells. For alleviating uplink interference caused by co-channel femto users to macrocells, a distributed femtocell power control algorithm is developed based on non-cooperative game theory in [5], while in [6] femto users are priced for causing interference to macrocells in the power allocation based on a Stackelberg model. In [7], cross-tier interference is mitigated through both open-loop and closed-loop uplink power controls. In [8], a distributed power control scheme is proposed based on a supermodular game.

A lot of work has also been done on subchannel allocation in co-channel deployment of femtocells. In [9], a hybrid frequency assignment scheme is proposed for femtocells deployed within coverage of a macrocell. In [10], distributed channel selection schemes are proposed for femtocells to avoid inter-cell interference, at the cost of reduced frequency reuse efficiency. In [11], a subchannel allocation algorithm based on a potential game

\section{照 Springer}

(c) 2012 Zhang et al; licensee Springer. This is an Open Access article distributed under the terms of the Creative Commons Attribution License (http://creativecommons.org/licenses/by/2.0), which permits unrestricted use, distribution, and reproduction in any medium, provided the original work is properly cited. 
model is proposed to mitigate both co-tier and cross-tier interferences.

Recently, several studies considering both power and subchannel allocation in femtocells have been reported. In [12], a joint power and subchannel allocation algorithm is proposed to maximize the total capacity of densely deployed femtocells, but the interference caused by femtocells to macrocells is not considered. In the collaborative resource allocation scheme [13], cross-tier interference is approximated as additive white Gaussian noise (AWGN). In the Lagrangian dual decomposition-based resource allocation scheme [14], constraints on cross-tier interference are used in power allocation, but subchannels are assigned randomly to femto users. In [15], a distributed downlink resource allocation scheme based on a potential game and convex optimization is proposed to increase the total capacity of macrocells and femtocells, but at the price of reduced femtocell capacity. In [16], the distributed power and subchannel allocation for co-channel deployed femtocells is modeled as a non-cooperative game, for which a Nash Equilibrium is obtained based on a timesharing subchannel allocation, but the constraint on maximum femto-user transmit power is ignored in solving the non-cooperative game.

In this article, we focus on the uplink power and subchannel allocation problem of orthogonal frequency division multiple access (OFDMA)-based femtocells in cochannel deployment with macrocells. We first model the uplink power and subchannel allocation in femtocells as a non-cooperative game, where inter-cell interference is taken into account in maximizing femtocell capacity and uplink interference from femto users to the macrocell is alleviated by charging each femto user a price proportional to the amount of interference that it causes to the macrocell. Based on the non-cooperative game, we then devise a semi-distributed algorithm for each femtocell to first assign subchannels to femto users and then allocate power to subchannels accordingly. Simulation comparisons with existing unpriced subchannel assignment and modified iterative water filling (MIWF)-based power allocation algorithms show that the proposed interferenceaware femtocell uplink resource allocation algorithm is able to provide improved capacities for not only femtocells, but also the macrocell, as well as comparable or even better tiered fairness in a co-channel two-tier network.

The rest of this article is organized as follows. The system model and problem formulation are presented in "System model and problem formulation" section. In "Interference-aware resource allocation" section, the interference-aware femtocell uplink resource allocation algorithm is proposed. Performance of the proposed algorithm is evaluated by simulations in "Simulation results and discussion" section. Finally, "Conclusion" section concludes the article.

\section{System model and problem formulation System model}

As shown in Figure 1, we consider a two-tier OFDMA network where $K$ co-channel FBSs are randomly overlaid on a macrocell. We focus on resource allocation in the uplink of femtocells, that is, the subchannel assignment to femto users and the power allocation on subchannels in femtocells. Let $M$ and $F$ denote the numbers of active macro users camping on the macrocell and active femto users camping on each femtocell, respectively. Users are uniformly distributed in the coverage area of their serving cell. All femtocells are assumed to be closed access [17]. The OFDMA system has a bandwidth of $B$, which is divided into $N$ subchannels. Channel fading on each subcarrier is assumed the same within a subchannel, but may vary across different subchannels. We assume that channel fading is composed of path loss and frequency-flat Rayleigh fading.

We denote $g_{k, u, n}^{\mathrm{MF}}$ and $g_{j, k, u, n}^{\mathrm{FF}}$ as the channel gains on subchannel $n$ from femto user $u$ in femtocell $k$ to the MBS and FBS $j$, respectively, where $j, k \in\{1,2, \ldots, K\}, u \in$ $\{1,2, \ldots, F\}$, and $n \in\{1,2, \ldots, N\}$; denote $g_{w, n}^{\mathrm{M}}$ and $g_{k, w, n}^{\mathrm{FM}}$ as the channel gains on subchannel $n$ from macro user $w(\in\{1,2, \ldots, M\})$ to the MBS and FBS $k$, respectively; denote $p_{k, u, n}^{\mathrm{F}}$ and $p_{w, n}^{\mathrm{M}}$ as the transmit power levels on subchannel $n$ of femto user $u$ in femtocell $k$ and macro user $w$, respectively. Then, we define $\boldsymbol{P}_{n}=\left[p_{k, u, n}^{\mathrm{F}}\right]_{K \times F}$ as the power allocation matrix of the $K$ femtocells on subchannel $n$, and $\boldsymbol{A}_{n}=\left[a_{k, u, n}\right]_{K \times F}$ as the subchannel assignment indication matrix for the $K$ femtocells on subchannel $n$, where $a_{k, u, n}=1$ if subchannel $n$ is assigned to femto user $u$ in femtocell $k$, and $a_{k, u, n}=0$ otherwise.

The received signal-to-interference and noise ratio (SINR) for femto user $u$ on the $n$th subchannel in the $k$ th femtocell is given by

$$
\gamma_{k, u, n}^{\mathrm{F}}=\frac{a_{k, u, n} p_{k, u, n}^{\mathrm{F}} g_{k, k, u, n}^{\mathrm{FF}}}{\sum_{(j, v) \neq(k, u)}^{(K, F)} a_{j, v, n} p_{j, v, n}^{\mathrm{F}} g_{k, j, v, n}^{\mathrm{FF}}+p_{w, n}^{\mathrm{M}} g_{k, w, n}^{\mathrm{FM}}+\sigma^{2}}
$$

where $\sum_{(j, v) \neq(k, u)}^{(K, F)} a_{j, v, n} p_{j, v, n}^{\mathrm{F}} g_{k, j, v, n}^{\mathrm{FF}}=\sum_{j=1}^{K} \sum_{v=1}^{F} a_{j, v, n} p_{j, v, n}^{\mathrm{F}}$ $g_{k, j, v, n}^{\mathrm{FF}}-a_{k, u, n} p_{k, u, n}^{\mathrm{F}} g_{k, k, u, n}^{\mathrm{FF}}$ is the interference caused by other co-channel femtocells, $p_{w, n}^{\mathrm{M}} g_{k, w, n}^{\mathrm{FM}}$ is the interference caused by the macrocell, and $\sigma^{2}$ is the AWGN power.

The SINR for macro user $w$ using the $n$th subchannel is given by

$$
\gamma_{w, n}^{\mathrm{M}}=\frac{p_{w, n}^{\mathrm{M}} g_{w, n}^{\mathrm{M}}}{\sum_{j=1}^{K} \sum_{v=1}^{F} a_{j, v, n} p_{j, v, n}^{\mathrm{F}} g_{j, v, n}^{\mathrm{MF}}+\sigma^{2}}
$$

Based on Shannon's capacity formula, the capacities on subchannel $n$ of femto user $u$ in femtocell $k$ and macro 
Chang et al. EURASIP Journal on Wireless Communications and Networking 2012, 2012:289

Page 3 of 9

http://jwcn.eurasipjournals.com/content/2012/1/289

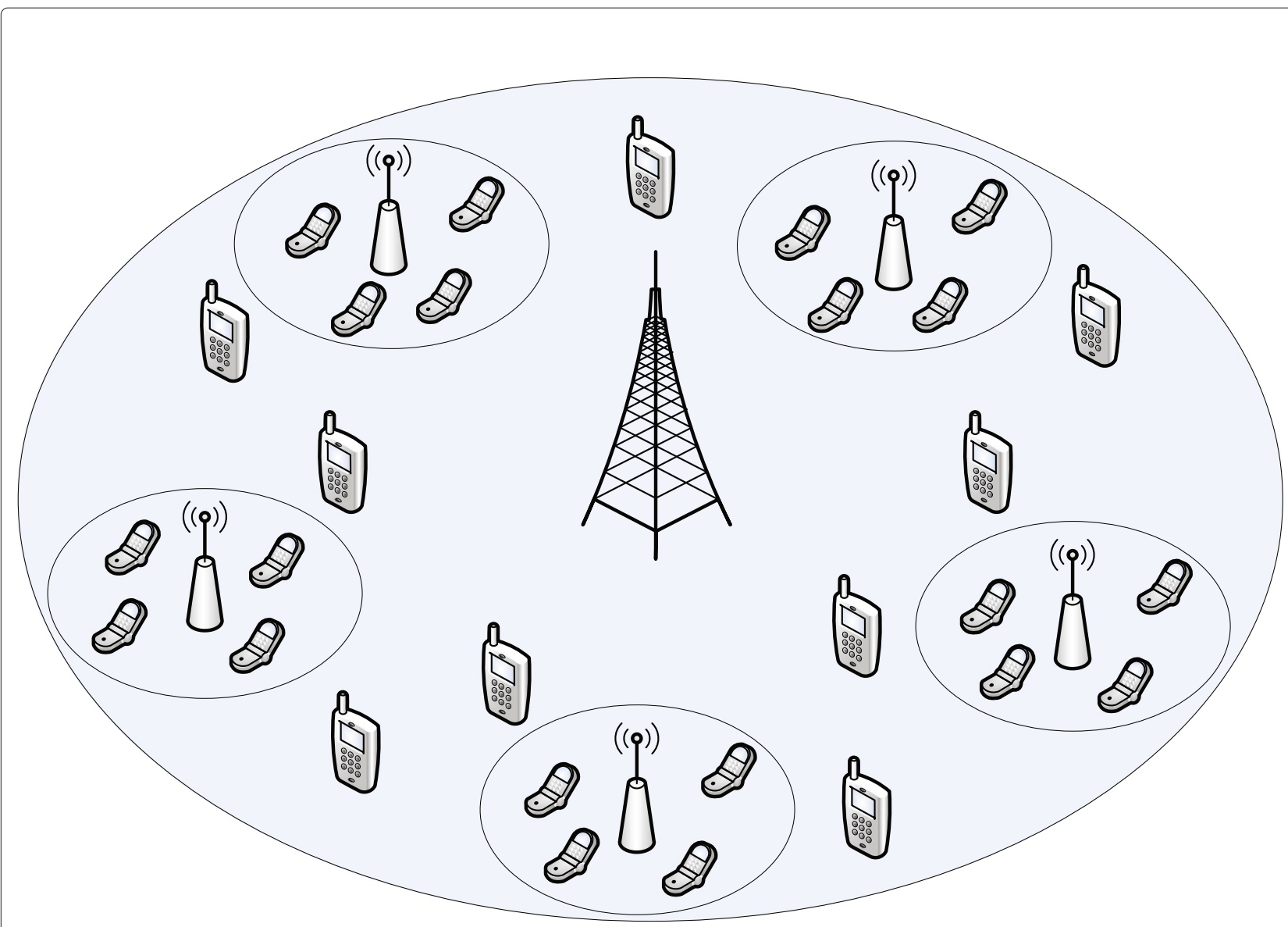

Figure 1 Topology of the two-tier network comprising by a macrocell and $K$ co-channel femtocells.

user $w$ are given, respectively, by

$$
\begin{aligned}
& C_{k, u, n}^{\mathrm{F}}=\frac{B}{N} \log _{2}\left(1+\gamma_{k, u, n}^{\mathrm{F}}\right) \\
& C_{w, n}^{\mathrm{M}}=\frac{B}{N} \log _{2}\left(1+\gamma_{w, n}^{\mathrm{M}}\right)
\end{aligned}
$$

Problem formulation

The maximization of the total capacity of the $K$ femtocells is formulated as follows.

$$
\begin{aligned}
& \max \sum_{k=1}^{K} \sum_{u=1}^{F} \sum_{n=1}^{N} C_{k, u, n}^{\mathrm{F}} \\
& \text { s.t. }\left\{\begin{array}{l}
\sum_{n=1}^{N} a_{k, u, n} p_{k, u, n}^{\mathrm{F}} \leq p^{\mathrm{max}}, \forall k, u \\
p_{k, u, n}^{\mathrm{F}} \geq 0, \forall k, u, n \\
a_{k, u, n} \in\{0,1\}, \forall k, u, n \\
\sum_{u=1}^{F} a_{k, u, n} \in\{0,1\}, \forall k, n
\end{array}\right.
\end{aligned}
$$

where a femto user's total transmit power is constrained by $p^{\max }$, the power allocated to each subchannel is nonnegative, and each subchannel is assigned to no more than one user per femtocell.

It is assumed that the user assignment and power allocaton can be performed independently for each subchannel, then the maximization of the total capacity of $K$ femtocells is equivalent to the maximization of the total capacity of the $K$ femtocells on one subchannel, and (5) and (6) can be simplified to

$$
\begin{aligned}
& \max \sum_{k=1}^{K} \sum_{u=1}^{F} C_{k, u, n}^{\mathrm{F}}, \forall n \\
& \text { s.t. }\left\{\begin{array}{cc}
p_{k, u, n}^{\mathrm{F}} \leq p_{n}^{\max }, & \forall k, u, n \\
p_{k, u, n}^{\mathrm{F}} \geq 0, & \forall k, u, n \\
a_{k, u, n} \in\{0,1\}, & \forall k, u, n \\
F & \forall k, n \\
\sum_{u=1}^{F} a_{k, u, n} \in\{0,1\}, &
\end{array}\right.
\end{aligned}
$$


where $p_{n}^{\max }$ is the transmit power constraint on subchannel $n$ for a femto user. Without loss of generality, we assume that $p_{n}^{\max }=p^{\max } / n$.

\section{Interference-aware resource allocation}

In this section, we first model the uplink power and subchannel allocation problem in femtocells using a noncooperative game theory framework $[18,19]$, where a pricing scheme is imposed on femto users to mitigate the uplink interference caused by femto users to the macrocell. Then based on the non-cooperative game framework, we propose a semi-distributed algorithm for femtocells to assign subchannels to femto users assuming an arbitrary power allocation, and then optimize the power allocation on subchannels based on the obtained subchannel assignment.

\section{A game theoretic framework}

Based on the microeconomic theory [20], we model the femtocell uplink resource allocation problem as a femtocell non-cooperative resource allocation game (FNRAG). The $K$ femtocells are considered as selfish, rational players. Each of them tries to maximize its utility without considering the impact on other players. The FNRAG for subchannel $n$ can be expressed as

$$
\boldsymbol{G}_{n}=\left\langle\boldsymbol{K},\left\{\boldsymbol{A}_{n}, \boldsymbol{P}_{n}\right\}, \boldsymbol{\mu}_{n}^{c}\right\rangle, \forall n
$$

where $\boldsymbol{K}=\{1, \ldots, k, \ldots, K\}, \forall n \in\{1,2, \ldots, N\}$ is the set of femtocells playing the game; $\left\{\boldsymbol{A}_{n}, \boldsymbol{P}_{n}\right\}$ is the strategy space of the players, with $\boldsymbol{A}_{n}$ and $\boldsymbol{P}_{n}$ being the subchannel assignment space and the power allocation strategy space, respectively; and $\boldsymbol{\mu}_{n}^{c}=\left\{\mu_{1, n}^{c}, \mu_{2, n}^{c}, \ldots, \mu_{K, n}^{c}\right\}$ is the set of net utility functions of the $K$ players, in which

$$
\mu_{k, n}^{c}=\sum_{u=1}^{F}\left(C_{k, u, n}^{\mathrm{F}}-\alpha a_{k, u, n} g_{k, u, n}^{\mathrm{MF}} p_{k, u, n}^{\mathrm{F}}\right)
$$

where $\alpha\left(\in \mathbb{R}^{+}\right)(\mathrm{bps} / \mathrm{W})$ is the pricing factor, and the price charged on a femto user is proportional to the uplink interference that it causes to the macrocell. If without the pricing part in the utility function, then a player will tend to maximize its utility by using the maximum transmit power, because $C_{k, u, n}^{\mathrm{F}}$ monotonically increases with $p_{k, u, n}^{\mathrm{F}}$ according to (1) and (3). This will lead to severe uplink interference to the macrocell.

Given the power and subchannel allocation in all other co-channel femtocells, the net utility function of femtocell $k$ can be rewritten as

$$
\begin{aligned}
& \mu_{k, n}^{c}\left(\boldsymbol{p}_{k, n}, \boldsymbol{a}_{k, n} \mid \boldsymbol{P}_{-k, n}, \boldsymbol{A}_{-k, n}\right) \\
& =\sum_{u=1}^{F}\left[\frac{B}{N} \log _{2}\left(1+\frac{a_{k, u, n} p_{k, u, n}^{\mathrm{F}} g_{k, k, u, n}^{\mathrm{FF}}}{I_{k, u, n}}\right)-\alpha a_{k, u, n} g_{k, u, n}^{\mathrm{MF}} p_{k, u, n}^{\mathrm{F}}\right]
\end{aligned}
$$

where $\boldsymbol{p}_{k, n}=\left\{p_{k, 1, n}^{\mathrm{F}}, p_{k, 2, n}^{\mathrm{F}}, \ldots, p_{k, F, n}^{\mathrm{F}}\right\}, \quad \boldsymbol{a}_{k, n}=$ $\left\{a_{k, 1, n}, a_{k, 2, n}, \ldots, a_{k, F, n}\right\}, \boldsymbol{P}_{-k, n}$ is the $(K-1) \times F$ matrix obtained by removing the $k^{\text {th }}$ row from $\boldsymbol{P}_{n}, \boldsymbol{A}_{-k, n}$ is the $(K-1) \times F$ matrix obtained by removing the $k$ th row from $\boldsymbol{A}_{n}$, and $I_{k, u, n}=\sum_{(j, v) \neq(k, u)}^{(K, F)} a_{j, v, n} p_{j, v, n}^{\mathrm{F}} g_{k, j, v, n}^{\mathrm{FF}}+p_{w, n}^{\mathrm{M}} g_{k, w, n}^{\mathrm{FM}}+\sigma^{2}$.

Definition 1. Given the uplink power allocation and subchannel assignment of all other co-channel femtocells, the best response of femtocell $k$ is given by

$$
\left(\hat{\boldsymbol{p}}_{k, n}, \hat{\boldsymbol{a}}_{k, n}\right)=\arg \max _{\boldsymbol{p}_{k, n}, \boldsymbol{a}_{k, n}} \mu_{k, n}^{c}\left(\boldsymbol{p}_{k, n}, \boldsymbol{a}_{k, n} \mid \boldsymbol{P}_{-k, n}, \boldsymbol{A}_{-k, n}\right), \forall k
$$

In the following sections, in order to solve the noncooperative femtocell uplink resource allocation game in a semi-distributed manner, we devise a semi-distributed algorithm for each femtocell to first assign subchannels to its femto users for given power and subchannel allocation in all other femtocells and assuming an arbitrary power allocation of its own, and then optimize the power allocation based on the obtained subchannel assignment.

\section{Interference-aware subchannel allocation}

In this section, we improve the subchannel allocation method in $[21,22]$ by pricing femto users according to their interference to macrocell in femtocell subchannel allocation.

Assuming that the $k$ th row of the matrix $\boldsymbol{P}_{n}$ contains an arbitrary power allocation of femtocell $k$ on subchannel $n$, and given the power allocation and subchannel assignment of all other femtocells on subchannel $n$, then the best assignment of subchannel $n$ in femtocell $k$ is given by

$$
\hat{\boldsymbol{a}}_{k, n}=\arg \max _{\boldsymbol{a}_{k, n}} \mu_{k, n}^{c}\left(\boldsymbol{a}_{k, n} \mid \boldsymbol{P}_{n}, \boldsymbol{A}_{-k, n}\right), \quad \forall k
$$

According to (8), at most one element of $\boldsymbol{a}_{k, n}$ can take value of 1 . Therefore, based on (11), the problem in (13) is equivalent to

$$
\begin{array}{r}
\hat{u}_{k, n}=\arg \max _{u}\left[\frac{B}{N} \log _{2}\left(1+\frac{p_{k, u, n}^{\mathrm{F}} g_{k, k, u, n}^{\mathrm{FF}}}{I_{k, u, n}}\right)\right. \\
\left.-\alpha g_{k, u, n}^{\mathrm{MF}} p_{k, u, n}^{\mathrm{F}}\right], \quad \forall k, n
\end{array}
$$

where $\hat{u}_{k, n}$ is the best user for channel $n$ in femtocell $k$ to assign, and the assignment of subchannel $n$ in femtocell $k$ is indicated by $\hat{\boldsymbol{a}}_{k, n}=\left\{\hat{a}_{k, 1, n}, \hat{a}_{k, 2, n}, \ldots, \hat{a}_{k, F, n}\right\}$, where

$$
\hat{a}_{k, u, n}=\left\{\begin{array}{l}
1, \text { if } u=\hat{u}_{k, n}, \\
0, \text { otherwise. }
\end{array}\right.
$$


In order to remove the dependence of the subchannel assignment on the assumed arbitrary power allocation, we let $\hat{\gamma}_{k, n}=\max _{u} \frac{p_{k, u, n}^{\mathrm{F}} g_{k, k, u, n}^{\mathrm{FF}}}{I_{k, u, n}}$, and then the transmit power of femto user $u$ corresponding to $\hat{\gamma}_{k, n}$ is given by $\frac{\hat{\gamma}_{k, n} I_{k, u, n}}{g_{k, k, u, n}^{\mathrm{F}}}$. Accordingly, Equation (14) can be rewritten as

$$
\begin{aligned}
\hat{u}_{k, n} & =\arg \max _{u}\left[\frac{B}{N} \log _{2}\left(1+\hat{\gamma}_{k, n}\right)-\alpha g_{k, u, n}^{\mathrm{MF}} \frac{\hat{\gamma}_{k, n} I_{k, u, n}}{g_{k, k, u, n}^{\mathrm{FF}}}\right]_{, \forall k, n} \\
& =\arg \min _{u} \frac{g_{k, u, n}^{\mathrm{MF}}}{g_{k, k, u, n}^{\mathrm{FF}}} I_{k, u, n}
\end{aligned}
$$

\section{Interference-aware power allocation}

Once the uplink subchannel assignment has been determined by using (15) and (16) in each femtocell, the FNRAG in (9) can be reduced to a femtocell noncooperative power allocation game (FNPAG): $\boldsymbol{G}_{n}{ }^{\prime}=$ $\left\langle\boldsymbol{K}, \boldsymbol{P}_{n}, \boldsymbol{\mu}_{n}^{c}\right\rangle, \forall n$. Since subchannels have been assigned to specific femto users in each cell, we will drop the subscript $u$ for simplicity hereafter.

Definition 2. Denote $\hat{\boldsymbol{p}}_{n}=\left\{\hat{p}_{1, n}^{\mathrm{F}}, \hat{p}_{2, n}^{\mathrm{F}}, \ldots, \hat{p}_{K, n}^{\mathrm{F}}\right\}$ as the optimal transmit power vector of the $K$ co-channel femto users allocated to subchannel $n$ under Nash Equilibrium in the FNPAG $\boldsymbol{G}_{n}{ }^{\prime}$, if

$$
\mu_{k, n}^{c}\left(\hat{p}_{k, n}^{\mathrm{F}} \mid \hat{\boldsymbol{p}}_{-k, n}, \boldsymbol{A}_{n}\right) \geq \mu_{k, n}^{c}\left(p_{k, n}^{\mathrm{F}} \mid \hat{\boldsymbol{p}}_{-k, n}, \boldsymbol{A}_{n}\right), \quad \forall p_{k, n}^{\mathrm{F}} \geq 0
$$

where $\hat{\boldsymbol{p}}_{-k, n}=\left\{\hat{p}_{1, n}^{\mathrm{F}}, \ldots, \hat{p}_{k-1, n}^{\mathrm{F}}, \hat{p}_{k+1, n}^{\mathrm{F}}, \ldots, \hat{p}_{K, n}^{\mathrm{F}}\right\}$ is the optimal transmit power vector of the $K-1$ co-channel femto users using subchannel $n$ under Nash Equilibrium except for the co-channel femto user in femtocell $k$, and Nash Equilibrium is defined as the fixed points where no player can improve its utility by changing its strategy unilaterally [20].

Theorem 1. A Nash Equilibrium exists in the FNPAG: $\boldsymbol{G}_{n}{ }^{\prime}=\left\langle\boldsymbol{K}, \boldsymbol{P}_{n}, \boldsymbol{\mu}_{n}^{c}\right\rangle, \quad \forall n$.

Proof. According to [20], a Nash Equilibrium exists in $\boldsymbol{G}_{n}{ }^{\prime}$ if the following two conditions are satisfied:

(1) $\boldsymbol{P}_{n}$ is non-empty, convex and compact in the finite Euclidean space $\Re^{K \times F}$.

(2) $\boldsymbol{\mu}_{n}^{c}$ is continuous and concave with respect to $\boldsymbol{P}_{n}$.

Since the power allocated on each subchannel is constrained between zero and the maximum power $p_{n}^{\max }$, the power allocation matrix $\boldsymbol{P}_{n}$ is convex and compact, and condition (1) is satisfied.

For condition (2), it can be seen from (11) that $\boldsymbol{\mu}_{n}^{c}$ is continuous with respect to $\boldsymbol{P}_{n}$. To prove the quasi-concave

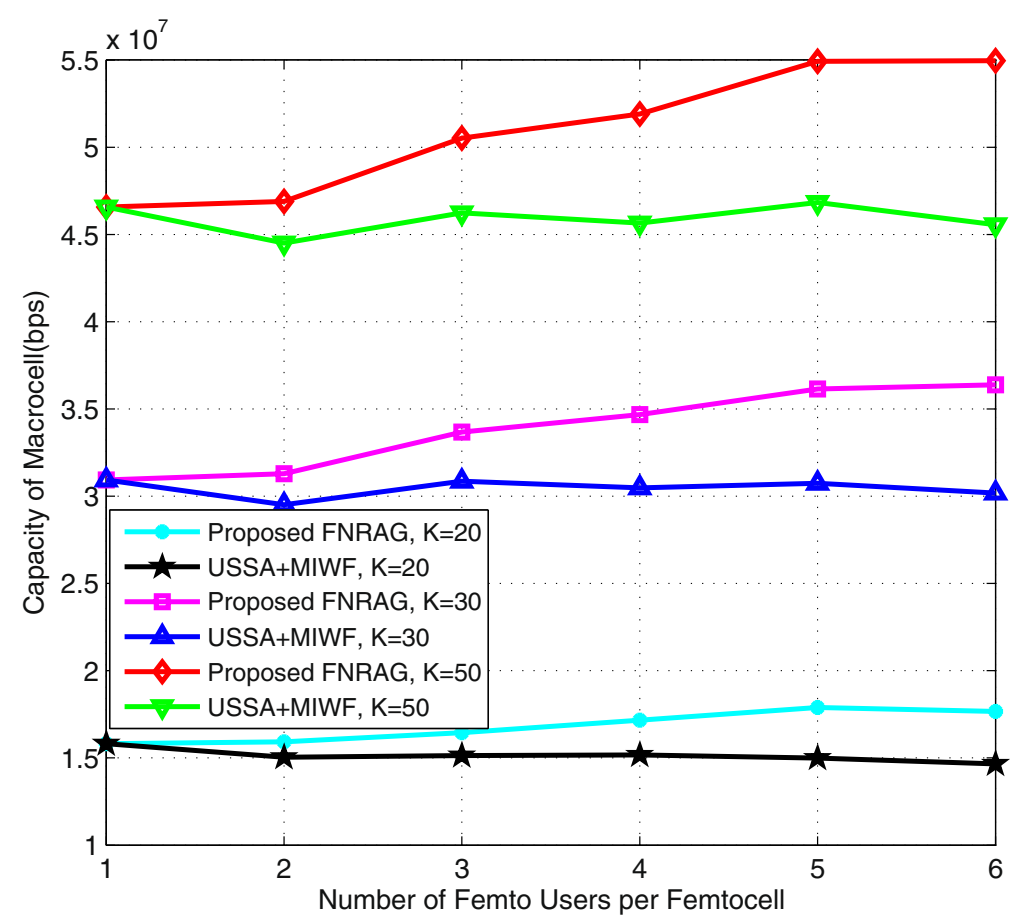

Figure 2 Capacity of macrocell versus the number of femto users $F$ in each femtocell with the number of macro users $M=50$. 
property of (11), we take the derivative of (11) with respect to $p_{k, n}^{\mathrm{F}}$, and get

$$
\frac{\partial \mu_{k, n}^{c}}{\partial p_{k, n}^{\mathrm{F}}}=\frac{B}{N \ln 2} \frac{g_{k, k, n}^{\mathrm{FF}}}{\left(I_{k, n}+p_{k, n}^{\mathrm{F}} g_{k, k, n}^{\mathrm{FF}}\right)}-\alpha g_{k, n}^{\mathrm{MF}}
$$

Taking the second-order derivative of (11) with respect to $p_{k, n}^{\mathrm{F}}$ yields

$$
\frac{\partial^{2} \mu_{k, n}^{c}}{\partial^{2} p_{k, n}^{\mathrm{F}}}=-\frac{B}{N \ln 2} \frac{\left(g_{k, k, n}^{\mathrm{FF}}\right)^{2}}{\left(I_{k, n}+p_{k, n}^{\mathrm{F}} g_{k, k, n}^{\mathrm{FF}}\right)^{2}} \leq 0
$$

Therefore, $\mu_{k, n}^{c}$ is a quasi-concave function of $p_{k, n}^{\mathrm{F}}$. Since both conditions (1) and (2) hold, a Nash Equilibrium exists in the FNPAG. This completes the proof.

Lemma 1. The best response of femtocell $k$ to the FNPAG $\boldsymbol{G}_{n}{ }^{\prime}$ is given by

$$
\begin{aligned}
& \hat{\boldsymbol{p}}_{k, n}=\hat{p}_{k, n}^{\mathrm{F}} \hat{\boldsymbol{a}}_{k, n} \\
& \hat{p}_{k, n}^{\mathrm{F}}=\left[\frac{B}{N \ln 2} \cdot \frac{1}{\alpha g_{k, n}^{\mathrm{MF}}}-\frac{I_{k, n}}{g_{k, k, n}^{\mathrm{FF}}}\right]_{0}^{p_{n}^{\max }}
\end{aligned}
$$

where $[x]_{a}^{b}=\min \{\max \{a, x\}, b\}$.

Proof. The $\hat{p}_{k, n}^{\mathrm{F}}$ in (21) is obtained by setting (18) to zero and solving the resulting equation for $p_{k, n}^{\mathrm{F}}$.

Since (21) should be non-negative, and the interference price factor $\alpha$ is non-negative too, we get

$$
0 \leq \alpha \leq \frac{B}{N \ln 2} \cdot \frac{g_{k, k, n}^{\mathrm{FF}}}{g_{k, n}^{\mathrm{MF}} I_{k, n}}
$$

Theorem 2. The FNPAG has a unique Nash Equilibrium.

Proof. It can be proved following similar proof in [5,23].

\section{Semi-distributed implementation}

Since only local information, such as uplink interference and channel gains seen by femto users, is needed for calculating (16) and (21), the interference-aware femtocell uplink subchannel allocation scheme and power allocation scheme proposed in "Interference-aware subchannel allocation" and "Interference-aware power allocation" sections can be implemented in a distributed and semi-distributed manners, respectively, as outlined in Algorithm 1.

Note that, $g_{k, j, v, n}^{\mathrm{FF}}$ and $g_{k, w, n}^{\mathrm{FM}}$ for the uplink can be estimated at femto user $u$ in femtocell $k$ by measuring the downlink channel gain of subchannel $n$ from femtocell $j$ and the macrocell, respectively, and utilizing the symmetry between uplink and downlink channels, or by using the site specific knowledge [5]. Furthermore, it can be assumed that there is a direct wire connection between an FBS and the MBS for the FBS to coordinate with the central MBS $[4,6]$, according to a candidate scheme proposed for 3GPP HeNB mobility enhancement [24].

Algorithm 1 can be implemented by each FBS, who only utilizing local information and limited interaction with MBS, therefore, Algorithm 1 is semi-distributed and the practicability is guaranteed.

\section{Algorithm 1 Semi-distributed algorithm to solve FNRAG}

1: FBS set: $\mathcal{K}=\{1,2, \ldots, K\}$; Femto user set per femtocell: $\mathcal{F}=\{1,2, \ldots, F\}$.

2: Interference-Aware Subchannel Allocation

3: Allocate the same power to each subchannel;

4: Femto user $u$ in femtocell $k$ measures $g_{k, u, n}^{\mathrm{MF}}, g_{k, k, u, n}^{\mathrm{FF}}$ and $I_{k, u, n}, \forall k, u, n$

5: $a_{k, u, n}=0, \forall k, u, n$

6: for each FBS do

7: $\quad$ Subchannel set: $\mathcal{N}=\{1,2, \ldots, N\}$

8: $\quad$ for $u=1$ to $F$ do

9: $\quad$ (a) find $n^{*}=\arg \min _{n \in \mathcal{N}} \frac{g_{k, u, n}^{\mathrm{MF}}}{g_{k f, u, n}^{\mathrm{FF}}} I_{k, u, n}$;

10: $\quad$ (b) $a_{k, u, n^{*}}=1$;

11: $\quad$ (c) $\mathcal{N}=\mathcal{N}-\left\{n^{*}\right\}$;

12: end for

13: $\quad$ while $\mathcal{N} \neq \phi$ do

14: $\quad$ (a) find $\left(u^{*}, n^{*}\right)=\arg \min _{u \in \mathcal{F}, n \in \mathcal{N}} \frac{g_{k, u, n}^{\mathrm{MF}}}{g_{k, k, u, n}^{\mathrm{FF}}} I_{k, u, n}$;

15: $\quad$ (b) $a_{k, u^{*}, n^{*}}=1$;

16: $\quad$ (c) $\mathcal{N}=\mathcal{N}-\left\{n^{*}\right\}$;

17: end while

18: end for

19: Interference-Aware Power Allocation

20: for each FBS do

21: $\quad$ for $n=1$ to $N$ do

22: $\quad$ calculate (20) and (21);

23: $\quad$ end for

24: end for

\section{Simulation results and discussion}

In this section, we present simulation results to evaluate the performance of the proposed interference-aware FNRAG algorithm, as compared with the unpriced suboptimal subchannel allocation (USSA) and MIWF-based power allocation algorithm [4,25], which are outlined in Algorithm 2. Both the system capacity and the fairness between femto-tier and macro-tier are evaluated in the simulations. 


\section{Algorithm 2 USSA and MIWF algorithm}

1: FBS set: $\mathcal{K}=\{1,2, \ldots, K\}$; Femto user set per femtocell: $\mathcal{F}=\{1,2, \ldots F\}$.

2: USSA

3: Allocate the same power to each subchannel;

4: Femto user $u$ in femtocell $k$ measures $g_{k, k, u, n}^{\mathrm{FF}}$ and $I_{k, u, n}, \forall k, u, n$;

5: $a_{k, u, n}=0, \forall k, u, n$;

6: for $k=1$ to $K$ do

7: Subchannel set: $\mathcal{N}=\{1,2, \ldots, N\}$

8: $\quad$ for $u=1$ to $F$ do

9: $\quad$ (a) find $n^{*}=\arg \max _{n \in \mathcal{N}} \frac{g_{k, k, u, n}^{\mathrm{FF}}}{I_{k, u, n}}$;

10: $\quad$ (b) $a_{k, u, n^{*}}=1$;

11: $\quad$ (c) $\mathcal{N}=\mathcal{N}-\left\{n^{*}\right\}$

12: end for

13: while $\mathcal{N} \neq \phi$ do

14: $\quad$ (a) find $\left(u^{*}, n^{*}\right)=\arg \max _{u \in \mathcal{F}, \backslash \in \mathcal{N}} \frac{g_{k, k, u, n}^{\mathrm{FF}}}{I_{k, u, n}}$;

15: $\quad$ (b) $a_{k, u^{*}, n^{*}}=1$;

16: $\quad$ (c) $\mathcal{N}=\mathcal{N}-\left\{n^{*}\right\}$;

17: end while

18: end for

19: MIWF-Based Power Allocation

20: Implement the MIWF algorithm using bisection search [25].

In the simulations, the macrocell has a coverage radius of $500 \mathrm{~m}$. Each femtocell has a coverage radius of $10 \mathrm{~m}$. $K$ FBSs and 50 macro users are randomly distributed in the macrocell coverage area. The minimum distance between the MBS and a macro user (or an FBS) is $50 \mathrm{~m}$. The minimum distance between FBSs is $40 \mathrm{~m}$. Femto users are uniformly distributed in the coverage area of their serving femtocell. Both macro and femtocells employ a carrier frequency of $2 \mathrm{GHz}, B=10 \mathrm{MHz}$, and $N=50$. The AWGN variance is given by $\sigma^{2}=$ $\frac{B}{N} N_{0}$, where $N_{0}=-174 \mathrm{~d} B m / H z$. The Rayleigh-fading channel gains are modeled as unit-mean exponentially distributed random variables. The average channel gain (including pathloss and antenna gains) for indoor femto user and outdoor macro user are modeled as $\lambda d^{-4}$ and $\lambda d^{-3}$, respectively, where $\lambda=2 \times 10^{-4}$ [5]. Besides, $\alpha$ is selected as $4 \times 10^{4}$ using the try-and-error method through simulations. The maximum transmit powers of a femto user and a macro user are set as 20 and $30 \mathrm{dBm}$, respectively.

Figure 2 shows the capacity of the macrocell when the number of femto users per femtocell increases from 1 to 6 , for $K=20,30$, and 50 . It can be observed that the proposed interference-aware FNRAG algorithm outperforms the USSA and MIWF-based algorithm by up to a $23 \%$ increase in macrocell capacity. As the number of femtocells $K$ increases, the advantage of the FNRAG algorithm becomes more noticeable, because the increased uplink interference caused by femtocells to the macrocell can be effectively mitigated by the pricing scheme imposed on femto users in the FNRAG algorithm, but not by the unpriced USSA and MIWF-based algorithm. As the number of femto users increases, the potential interferers will be more because of the co-channel deployed femtocells, but the number of available channels is constant. Therefore, the proposed algorithm will be more and more superior compared with the USSA and MIWF-based algorithm.

Figure 3 shows the total capacity of $K$ femtocells and macrocell when the number of femto users per femtocell increases from 1 to 6 , for $K=20,30$, and 50. We can see that the proposed FNRAG algorithm improves the total capacity of femtocells and macrocell over the USSA and MIWF-based algorithm by $5-10 \%$ when the number of femto users per femtocell is larger than 3 . More gain is obtained as $K$ increases, indicating that the proposed interference-aware FNRAG algorithm can also effectively mitigate interference between neighboring femtocells, and hence is more applicable in dense deployment of co-channel femtocells than the USSA and MIWF-based algorithm. As the number of femto users increases, the co-tier interference between femtocells is more severe, the interference-aware subchannel assignment will be more effective in co-tier interference mitigation.

In order to evaluate the fairness between the macro tier and femto tier, we use the tiered fairness index (TFI) [26], which is defined as

$$
f_{\mathrm{T} F I}=\frac{\left(M \sum_{w=1}^{M} C_{w}^{\mathrm{M}}+F \sum_{k=1}^{K} \sum_{u=1}^{F} C_{k, u}^{\mathrm{F}}\right)^{2}}{(M+F K)\left[\sum_{w=1}^{M}\left(M C_{w}^{\mathrm{M}}\right)^{2}+\sum_{k=1}^{K} \sum_{u=1}^{F}\left(F C_{k, u}^{\mathrm{F}}\right)^{2}\right]}
$$

where $C_{w}^{\mathrm{M}}$ and $C_{k, u}^{\mathrm{F}}$ are the capacities of macro user $w$ and femto user $u$ in femtocell $k$, respectively.

Figure 4 compares the tiered fairness performance between the proposed FNRAG algorithm and the USSA and MIWF-based algorithm. It can be observed that the tiered fairness of the proposed FNRAG algorithm gets close to or becomes even better than that of the USSA and MIWF-based algorithm, as the number of femto users per femtocell goes beyond 3. This is because the proposed FNRAG algorithm alleviates the uplink interference generated by femto users to the macrocell by charging each femto user a price proportional to the interference that it causes to the macrocell, and the macrocell capacity and consequently the 


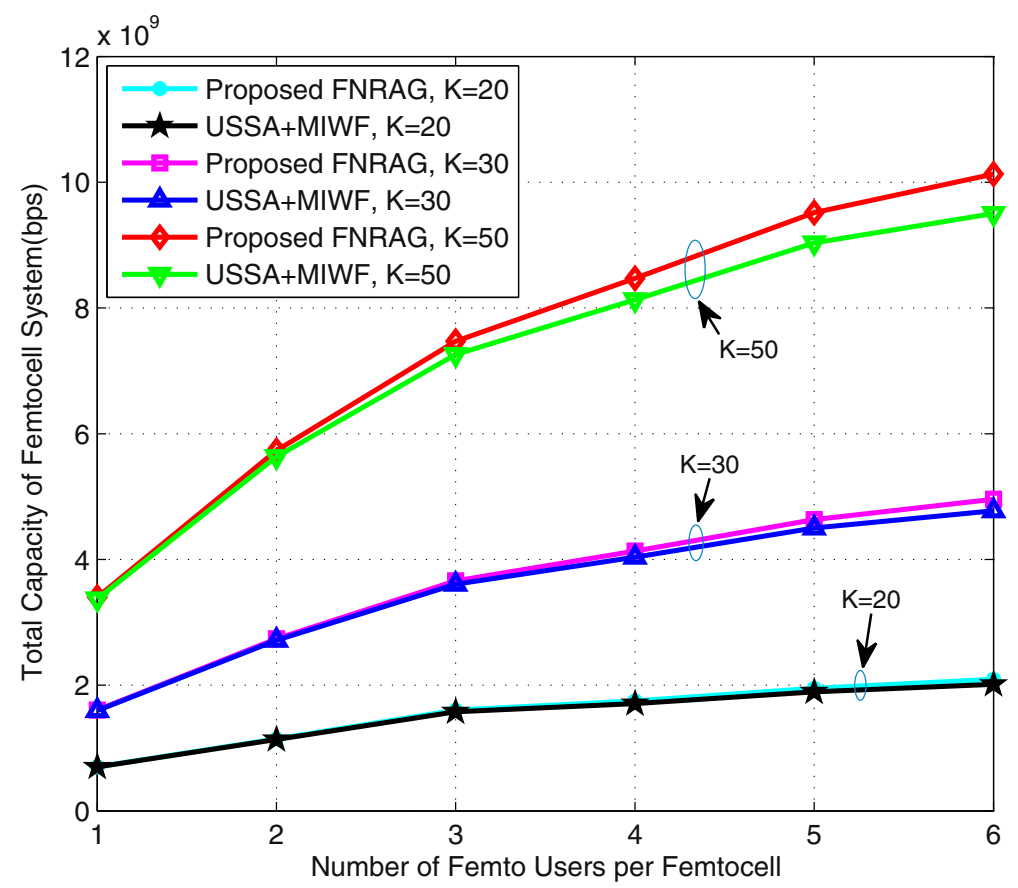

Figure 3 Capacity of macrocell and femtocells versus the number of femto users $F$ in each femtocell with the number of macro users $\mathbf{M}=\mathbf{5 0}$.

tiered fairness can be guaranteed. Since the USSA used in Algorithm 2 considers the fairness among femto users in each femtocell, the tiered fairness of Algorithm 2 is better than the proposed FNRAG algorithm when $F$ is less than 3. The tiered fairness improves as $K$ increases, because the spatial reuse gain increases with $K$.

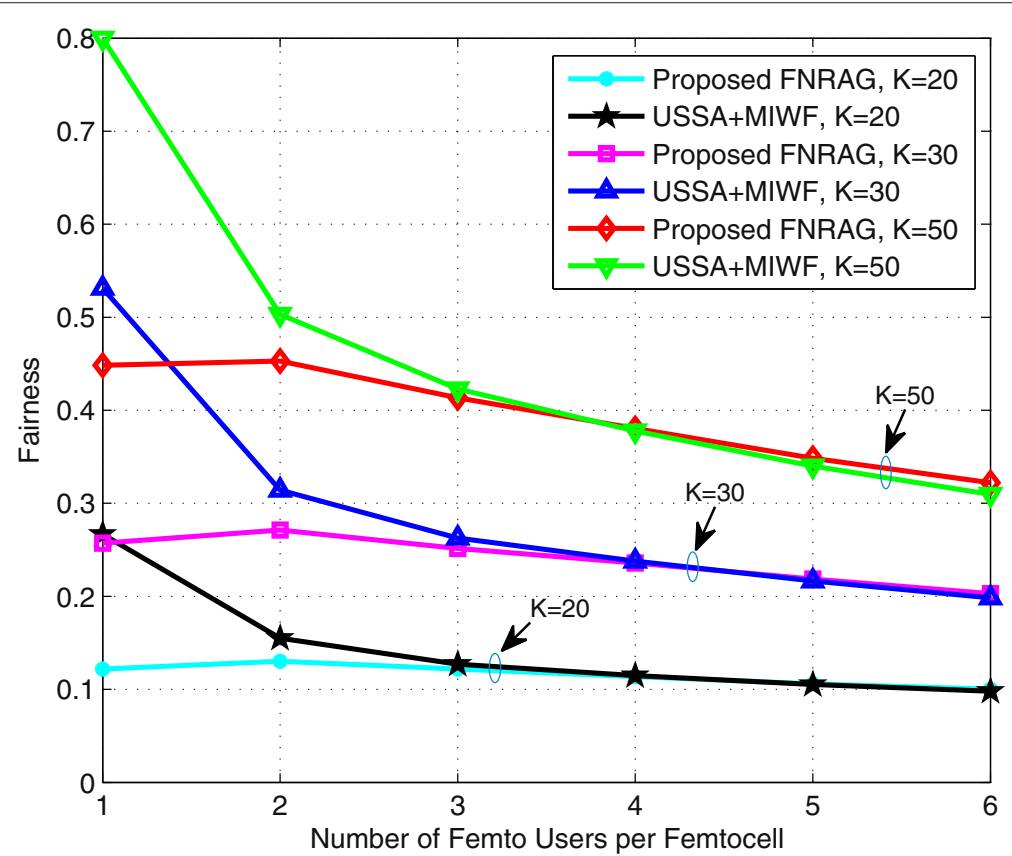

Figure 4 Tiered fairness versus the number of femto users $F$ in each femtocell with the number of macro users $\boldsymbol{M}=\mathbf{5 0}$. 


\section{Conclusion}

In this article, we have proposed a semi-distributed interference-aware resource allocation algorithm for the uplink of co-channel deployed femtocells, based on a noncooperative game framework. Using the proposed algorithm, each femtocell can maximize its capacity through resource allocation, taking into account inter-cell interference reported by its femto users, and with uplink femtoto-macro interference alleviated by a pricing scheme imposed on femto users. It has been shown through simulations that the proposed interference-aware resource allocation algorithm is able to provide improved capacities of both macrocell and femtocells, together with comparable tiered fairness, as compared with the existing unpriced subchannel allocation and MIWF-based power allocation algorithm.

\section{Endnote}

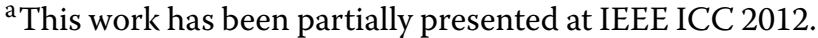

\section{Competing interest}

The authors declare that they have no competing interests.

\section{Acknowledgements}

The authors would like to thank Dr. David López-Pérez for his helpful discussions. This study was supported by the Sci-tech Projects of the Committee on Science and Technology of Beijing (D08080100620802, Z101101004310002), the National Natural Science Foundation of China (61101109), and the National Key Technology R\&D Program of China (2010ZX03003-001-01, 2011ZX03003-002-01). This study was also partially supported by the UK EPSRC Grants EP/H020268/1, CASE/CNA/07/106, and EP/G042713/1

\section{Author details}

${ }^{1}$ Beijing Key Laboratory of Network System Architecture and Convergence, Beijing University of Posts and Telecommunications, Beijing 100876, China. ${ }^{2}$ Institute of Telecommunications, King's College London, London WC2R 2LS, UK. ${ }^{3}$ The University of Sheffield, Sheffield S1 3JD, UK.

Received: 24 November 2011 Accepted: 24 August 2012 Published: 18 September 2012

\section{References}

1. D Lopez-Perez, A Valcarce, G de la Roche, J Zhang, OFDMA femtocells: a roadmap on interference avoidance. IEEE Commun. Mag. 47(9), 41-48 (2009)

2. 3GPP: E-UTRA and E-UTRAN overall description (2010)

3. V Chandrasekhar, J Andrews, A Gatherer, Femtocell networks: a survey. IEEE Commun. Mag. 46(9), 59-67 (2008)

4. K Son, S Lee, Y Yi, S Chong, REFIM: a practical interference management in heterogeneous wireless access networks. IEEE J. Sel. Areas Commun. 29(6), 1260-1272 (2011)

5. V Chandrasekhar, JG Andrews, T Muharemovic, Z Shen, A Gatherer, Power control in two-tier femtocell networks. IEEE Trans. Wirel. Commun. 8(8), 4316-4328 (2009)

6. X Kang, R Zhang, M Motani, Price-based resource allocation for spectrum-sharing femtocell networks: a Stackelberg game approach. IEEE J. Sel. Areas Commun. 30(3), 538-549 (2012)

7. HS Jo, C Mun, J Moon, JG Yook, Interference mitigation using uplink power control for two-tier femtocell networks. IEEE Trans. Wirel. Commun. 8(10), 4906-4910 (2009)

8. EJ Hong, SY Yun, DH Cho, Decentralized power control scheme in femtocell networks: a game theoretic approach. in IEEE PIMRC'09. (Tokyo, $1-5,2009)$
9. I Guvenc, MR Jeong, F Watanabe, H Inamura, A hybrid frequency assignment for femtocells and coverage area analysis for co-channe operation. IEEE Commun. Lett. 12(12), 880-882 (2008)

10. C Lee, JH Huang, LC Wang, Distributed channel selection principles for femtocells with two-tier interference. in IEEE 71st Vehicular Technology Conference (VTC 2010-Spring). (Taipei, 1-5, 2010)

11. I Mustika, K Yamamoto, H Murata, S Yoshida, Potential game approach for self-organized interference management in closed access femtocell networks. in IEEE 73rd Vehicular Technology Conference (VTC 2011-Spring). (Budapest, 1-5, 2011)

12. J Kim, DH Cho, A joint power subchannel allocation scheme maximizing system capacity in indoor dense mobile communication systems. IEEE Trans Veh. Technol. 59(9), 4340-4353 (2010)

13. K Lee, $\mathrm{H}$ Lee, $\mathrm{DH}$ Cho, Collaborative resource allocation for self-healing in self-organizing networks. in IEEE ICC'11. (Kyoto, 1-5, 2011)

14. J Zhang, Z Zhang, KWu, A Huang, Optimal distributed subchannel, rate and power allocation algorithm in OFDM-based two-tier femtocell networks. in IEEE VTC'10. (Taipei, 1-5, 2010)

15. L Giupponi, C Ibars, Distributed interference control in OFDMA-based femtocells. in IEEE 21th International Symposium on Personal, Indoor and Mobile Radio Communications (IEEE PIMRC'10). (Istanbul, 1201-1206, 2010)

16. JH Yun, KG Shin, Adaptive interference management of OFDMA femtocells for co-channel deployment. IEEE J. Sel. Areas Commun. 29(6)، 1225-1241 (2011)

17. S Yun, Y Yi, DH Cho, J Mo, Open or close: on the sharing of femtocells. in The 30th IEEE International Conference on Computer Communications (IEEE INFOCOM'11). (Shanghai, 116-120, 2011)

18. H Kwon, BG Lee, Distributed resource allocation through noncooperative game approach in multi-cell OFDMA systems. in IEEE International Conference on Communications (IEEE ICC'1 06). (Istanbul, 4345-4350, 2006)

19. Z Liang, YH Chew, CC Ko, On the modeling of a non-cooperative culticell OFDMA resource allocation game with integer bit-loading. in IEEE GLOBAL COMMUNICATIONS CONFERENCE (IEEE Globecom'09). (Hawai, 1-5, 2009)

20. D Fudenberg, J Tirole, Game Theory: (MIT Press, Cambridge, MA,1993)

21. CU Saraydar, NB Mandayam, DJ Goodman, Pricing and power control in a multicell wireless data network. IEEE J. Sel. Areas Commun. 19(10) 1883-1892 (2001)

22. F Wang, M Krunz, S Cui, Price-based spectrum management in cognitive radio networks. IEEE J. Sel. Topics Signal Process. 2, 74-87 (2008)

23. TC Tan, S Chuah, Tan, Fair subcarrier and power allocation for multiuser orthogonal frequency-division multiple access cognitive radio networks using a colonel blotto game. IET Commun. 5(11), 1607-1618 (2011)

24. 3GPP: Way forward proposal for (H)eNB to HeNB mobility (2010)

25. W Yu, Sum-capacity computation for the gaussian vector broadcast channel via dual decomposition. IEEE Trans. Inf. Theory. 52(2), 754-759 (2006)

26. MC Erturk, H Aki, I Guvenc, H Arslan, Fair and QoS-oriented spectrum splitting in macrocell-femtocell networks. in IEEE GLOBAL COMMUNICATIONS CONFERENCE (IEEE Globecom'10). (Miami, 1-6, 2010)

doi:10.1186/1687-1499-2012-289

Cite this article as: Zhang et al:: Resource allocation with interference mitigation in OFDMA femtocells for co-channel deployment ${ }^{\mathrm{a}}$. EURASIP Journal on Wireless Communications and Networking 2012 2012:289. 